\title{
Organizational Systematization Optimum Profitability by Systematic Management
}

\author{
Nasser Fegh-hi Farahmand* \\ Department of Industrial Management, College of Management, Economy and Accounting, Tabriz Branch, Islamic Azad University, \\ Tabriz, Iran \\ *Corresponding author: farahmand@iaut.ac.ir
}

Received July 19, 2014; Revised September 20, 2014; Accepted September 28, 2014

\begin{abstract}
Making a difference in organization or creating the very best product or service on the market or simply doing something loves to do. Furthermore, it seems that the emphasis on organizational empowerment as structure, tendency and systems has not yielded the desired results as some of the companies where these variables have been changed, after sometime, went back to experiencing declining performance. It is therefore obvious that more research needs to be done to identify characteristics that enhance organizational performance. There has been a longstanding bifurcation between the two with emotions labeled in pejorative terms and devalued in matters concerning the workplace. The form and structure of an organization's human resources system can affect employee motivation levels in several ways. Organizations can adopt various systematization optimum profitability humanistic approach empowerment practices to enhance employee satisfaction. This paper considers the systematic management. The strategic importance of workers is discussed and their interaction, as an asset, with other important organization assets. The basic methodologies for workers are then explained and their limitations are considered. The systematization optimum profitability revolution moves recording and analysis activities that were traditionally professional performance lines of activities focused to high operational content. The scientific and systematization optimum profitability progress, growth and internationalization of markets, processors are processes in which the accounting profession plays a leading role of systematic management.
\end{abstract}

Keywords: systematization optimum profitability management, systematization optimum profitability humanistic approach, systematization optimum profitability factor

Cite This Article: Nasser Fegh-hi Farahmand, “Organizational Systematization Optimum Profitability by Systematic Management.” Journal of Business and Management Sciences, vol. 2, no. 3 (2014): 69-78. doi: 10.12691/jbms-2-3-2.

\section{Introduction}

The operational concept based on customer satisfaction where the operation of quality management system is customer-oriented and aims at improving customer satisfaction; customers' needs and expectations are satisfied through clear management undertaking, communication, resource management and product realization process; the structure of measuring and monitoring customer satisfaction is proposed on the basis of overall performance of the quality system and requires enterprises evaluate performance from the perspective of customers. The form and structure of an organization's systematization optimum profitability humanistic approach can affect employee motivation levels in several ways. A discussion about a review on systematization optimum profitability humanistic approach in workplace has received relatively little attention from organizational technologic researchers. The first of the themes to be addressed concerns the relationship between emotion and rationality. Recognizing the importance of systematization optimum profitability humanistic approach in achieving flexibility in an international context expands the types of research questions related to the role of systematization optimum profitability humanistic approach functions in organizational performance, such as selection of human resources, training, and compensation and performance appraisal. Continuous training, employment security, performance appraisal and alternative compensation systematization can motivate skilled employees to engage in effective discretionary decision making and behavior in response to a variety of environmental contingencies $[1,4,11,19]$.

A discussion about a review on systematization optimum profitability humanistic approach in workplace has received relatively little attention from organizational behavior researchers. The first of the themes to be addressed concerns the relationship between emotion and rationality. There has been a longstanding bifurcation between the two with emotions labeled in pejorative terms and devalued in matters concerning the workplace.

The next theme explored centers around the theoretical grounding of emotion. Emotion is often described either in psychological terms as an individualized, intrapersonal response to some stimulus, or by contrast, a socially constituted phenomenon, depending upon the disciplinary 
perspective one adopts. This study has reviewed how organizations, as powerful culture eating institutions, have applied normative expectations and established boundaries for the acceptable expression of emotion among human resources system through tactics such as applicant screening and selection measures, employee training, offthe-job socialization opportunities, organizational rewards and the creation of rituals, ideologies and other symbols for indoctrinating the newly hired into the culture of the organization. There is no doubt that continuously such as brands, patents and workers lists makes a lot of sense rather than placing these organization critical assets in the accounting black hole known as goodwill. This study has reviewed how organizations, as powerful systematization optimum profitability humanistic approach empowerment and development eating institutions, have applied normative expectations and established boundaries for the acceptable expression of emotion among employees through tactics such as applicant screening and selection measures for [26,31,32,34,47]:

- Organizational empowerment by systematization optimum profitability humanistic approach empowerment,

- Systematization optimum profitability humanistic approach opportunities,

- Organizational empowerment by creation of systematization optimum profitability humanistic approach system,

- Organizational empowerment by creation of ideologies and other symbols for indoctrinating the newly hired into the culture of the organization,

- Organizational empowerment by creation of systematization optimum profitability humanistic approach empowerment for indoctrinating the strengthening of organization by systematization optimum profitability humanistic approach empowerment.

Organizational empowerment by human empowerment orientation is suggested to have a robust effect on individuals who endeavor to overcome the constrained commonplace conditions and deliver worthy achievements like social stability.

The principal weakness of the multiple excess earnings approach is that it is complicated to carry out. Furthermore, correctly identifying all the systematization optimum profitability humanistic approach operating functions and organizational systematization optimum profitability their respective functional returns and present values is open to distortion and inaccuracy due to the sensitivity of the valuation to key assumptions and source data. In the case of an acquisition, the excess returns will also include the value of any synergies resulting from the organization combination. In addition to its role in organizational survival funding, the organizational survival aggravation may serve as a strategic aggravation document for the entrepreneurs, a aggravation to guide the organizational survival and serve as a basis for taking strategic decisions and it may serve as a subsequent monitoring device.

\section{Systematization Optimum Profitability Humanistic Approach}

A positive experience throughout the workers cycle should foster trust and develop loyalty, therefore allowing an organization to generate more revenue for less incremental expenditure. However, the escalating cost of scientific and technological research and development, together with the limiting resources of governments, make such tendency of overall support impossible, even for relatively rich countries. The analysis of the systematization optimum profitability humanistic approach of the profession is conclusive to justify changes in training programs. Of all the professions, the organizational systematization optimum profitability is one of the most internationalized. The curricular reform of the curricula of public accounting have had a life too precarious because they are only temporary and pragmatic response to adjust the programs to the needs of the business environment and the demands dominant dogmatic rules of international accounting regulation changes.

In the international arena and global, there is a widely accepted framework to guide a global organizational systematization optimum profitability curriculum, consisting of six elements [3,5,22,28,33,40]:

- Organizational systematization optimum profitability knowledge and general skills,

- Organizational systematization optimum profitability detailed curriculum for technical,

- Organizational systematization optimum profitability professional examinations,

- Organizational systematization optimum profitability practical experience,

- Organizational systematization optimum profitability continuing professional education,

- Organizational systematization optimum profitability outlines certification.

The advancement of distance education models and online is irreversible if it is to take advantage of information systematization optimum profitability and communication, yet there is scant information and no evaluation indicators for organizational systematization optimum profitability control of tenders distance programs.

With regard to the existing supply in the market for accounting professionals' organizational systematization optimum profitability can find different levels of systematization optimum profitability and professional development by taking into account the programs and curricula in the various institutions of higher education. Capturing the wrong systematization optimum profitability humanistic approach information, unclear goals, inappropriate selection and use of systematization optimum profitability, inability to integrate workers and processes and use of misleading metrics or improper measurement approaches are the major barriers in implementing and managing human empowerment projects systematization that seek to identify individuals with the ability to learn and adapt to new situations and markets can provide a firm with competitive advantage. Systematization optimum profitability humanistic approach empowerment of organizational workers is defined as a complex feeling state accompanied by physiological arousal and overt behaviors. These words in essence, imply motion [10,12,17,27,41,48].

Human empowerment is typically functional because a motivated person moves himself towards some goal. But, human empowerment of organizational workers is primarily expressive because an emotional person is moved. Human empowerment of organizational workers 
can be motivating to the extent that human activity towards certain goal is influenced and sustained by feelings.

Whenever, try is to attain happiness or get rid of anger, irritation, etc. human empowerment of organizational workers plays significantly a motivated role. To the human empowerment of organizational workers aroused person, the most distinct aspect of emotion is the feeling component. For instance, a organizational worker becomes upset after every bang from his superior. This feeling cannot precisely be described. Perhaps, he may overcome this fear by developing another strong feeling that comforts him. The physiological component of human empowerment of organizational workers includes excitatory and inhibitory reactions that occur through arousal of the sympathetic nervous system. The sympathetic nervous system accelerates the heart, dilates the pupils, gives rise to adrenalin and controls the secretion of gastric juices. The individual becomes ready to fight or flight or affiliate.

The more we understand people and their total environment, the more their needs are likely to be met. When we talk about valuing workers relationships, the scope of definition is expansive. On the one hand, it is simply the value that workers generate for the organization. On the other hand, it is purely the value of the relationship. Neither definition is more correct than the other; however, the purpose and approach for valuing each are different.

A positive experience throughout the workers cycle should foster trust and develop loyalty, therefore allowing an organization to generate more revenue for less incremental expenditure. For example:

1) Happy existing workers are more willing to operation or services and try new operation or service offerings.

2) Making empower workers aware of operation and the cost of operation existing workers can be lower and, operation predicted.

This wide range of systematization optimum profitability humanistic approach programs of public accountant also has different characteristics competitive results when considering the transfer of knowledge, skills and values, development of professional practices, awareness of social responsibility performance in the environment. The higher systematic management is made up of public higher education institutions and private, which offer a diversified curriculum, relevant and linked to economic and social needs of the region from the technical to the doctoral level. Therefore, it requires the development of a systematization optimum profitability humanistic approach program with organizational systematization optimum profitability basis, technical, ethical and moral, with capacity for research, analysis of systematic management issues in various organization, national and international levels and low universally accepted standards [15,18,21,23,24].

This is to systematization optimum profitability professionals to use economic and organizational systematization optimum profitability information referring to the internal activities of organizations, management is developed in them, and in general, to various types of bodies falling under the operation of systematization optimum profitability humanistic approach as whole.

\section{Systematization Optimum Profitability Humanistic Approach Empowerment and Development}

The systematic management is highly sought. It started as an intern before finishing his career, allowing you to enter and have extensive experience in the professional field and then organizational systematization optimum profitability have large amounts of development within them. Contact with the client based on a thorough understanding of organizational systematization optimum profitability and systematization optimum profitability humanistic approach information, enables it to provide complete solutions and expanded in a portfolio of professional services. The comptroller has focused on the study of systematization optimum profitability humanistic approach planning and control of resources and verification operations in the organization to achieve the efficient use of the first and effectiveness in the latter, in order to achieve the objectives and goals set therein. Costs as an area of organizational systematization optimum profitability knowledge studies focused processes to identify, measure, collect, analyze and interpret the cost elements associated with the production and marketing of goods and services with the primary purpose of making decisions to achieve the objectives of organizational systematization optimum profitability established in the organization by systematic management. It is essential to meet the need of imparting organizational systematization optimum profitability knowledge to generate systematization optimum profitability information that, in turn serve to support both process management and leadership of organizations such as the requirements arising in the specific historical context in which these organizations are embedded.

Organizational systematization optimum profitability techniques and procedures applied in the identification, analysis, planning and cost control as a management tool and address. The organizational systematization optimum profitability is an important factor in entrepreneurship and enterprise development in general and systematization optimum profitability in particular, which enhances its importance and commitment of the profession to society. It promotes a program that encourages entrepreneurial generation of ideas for creating new businesses, achievable in terms of their own professional development goals in order to contribute to solving social, economic, political, cultural. Entrepreneurship and develop creative skills in systematization optimum profitability humanistic approach from the first cycle of training through contact with employers and advisory services to small businesses. It is perfectly able to form and run business, virtually no human activity that is dispensable resource use which must be managed and exploited in ways morally responsible for a professional in public accounting $[13,14,25,38,45]$.

The challenges that will face the professional in organizational systematization optimum profitability of the new millennium are large, uncertain and vague. 
Systematization optimum profitability humanistic approaches are one of the most valuable resources and organizations have to remain competitive. Modern organizations might achieve this by using organic systematization optimum profitability humanistic approach empowerment and development that promote the development of a human capital pool possessing a broad range of skills and that are able to engage in a wide variety of behavior. Systematization optimum profitability humanistic approach empowerment and development can be managed through conscious practices. This definition comes from an inter actionist approach, where, systematization optimum profitability humanistic approach empowerment are expressed in and partially determined by, the social environment. The systematization optimum profitability humanistic approach empowerment consists of frequency of interactions, attentiveness, variety of systematization optimum profitability humanistic approach empowerment required and dissonance. Systematization optimum profitability humanistic approach empowerment dissonance was discussed as a state where, in the emotions expressed are discrepant from the human development felt.

Job dissatisfaction and emotional exhaustion are proposed as outcomes of dissonance. This definition of emotional labor includes the organizational expectations for systematization optimum profitability humanistic approach in their inter actions with customers. According to systematization optimum profitability humanistic approach development regulation proposed the individual can regulate emotions at two points. At the first intervening point, an individual can engage in antecedentfocused human development regulation where, the individual modifies the situation or the perception of the situation in order to adjust human empowerment. It is stated that different types of antecedent-focused human development regulation by situation selection, situation modification, attention deployment and cognitive change. As integrationist theory discusses, people often choose the situations in which they act, including the situations that may create human development.

Systematization optimum profitability humanistic approach may choose their jobs, but for service employees there may be little opportunity for situation selection beyond that as a method to regulate development $[10,12,17,27,41,48]$.

To enact situation modification, an employee may choose to leave the work floor if a certain customer approaches, but this lack of availability is not quality customer service and may result in adverse consequences for the individual. With the lack of options to choose or modify the situation, human development regulation may take the form of the employee leaving the organization.

In short, systematization optimum profitability humanistic approach for development may not have the breadth of situation modification that is available outside of a work role. An individual could engage in responsefocused development regulation, or response modulation. In this process, the person has a tendency toward development and empowerment response, but manipulates how he or she shows that empowerment response by directly influencing physiological, experiential, or behavioral responding. Rather than adjusting the situation or the perception of the situation, the individual manipulates the empowerment expression of his or her reaction to the situation. This could be done with exercise or drugs that induce the appropriate state.

An individual may also adjust the intensity of the displayed emotion, or fake the expression entirely. Response-focused development and empowerment regulation corresponds with the process of surface acting. The job environment or a particular work event may induce an emotion response in the employee and behaviors may follow that would be inappropriate for the encounter. The chain of activities gives the products more added value than the sum of added values of all activities. It may be reasonable to suggest that it is the workers direct or indirect relationship with each of these activities that creates value for the organization.

Human empowerment and development as organizational ssupport activities, organizations tend to be highly decentralized and use informal means of coordination and control [26,31,32,34,47].

The reasons have to do with human bounded rationality. Bounded rationality refers to the fact that since human's Empowerment and development have not limited capacity, organizations can always find the absolute optimal solution by it. As all activities create value from and contribute to the workers relationship, it follows that the value of the organization and the value of the workers relationship could be considered to be the same. Expatriate managers are removed from the comfortable environment of their parental culture and placed in a less familiar culture. The systematization optimum profitability humanistic approach chain is often criticized as a dated framework that is only applicable to manufacturing industries and considers marketing in a silo rather than encompassing the whole enterprise. A management style that works at home may fail to produce the desired response abroad, or it may be even counterproductive.

\section{Systematic Management}

Systematization optimum profitability humanistic approach relationships appear to be similar; there are enough subtle differences to discount using brand value as a substitute for the value of a workers relationship. In contrast, there are operation drivers that cannot be attributed to the brand but can have a significant influence on the workers relationship with a organization.

Many organizations are becoming aware of the need to provide continued hands-on training rather than just predeparture awareness training. In contrast to pre-departure training, post-arrival training gives global managers a chance to evaluate their stressors after they have encountered them. Documentary and interpersonal training methods have additive benefits in preparing managers for intercultural systematization optimum profitability humanistic approach assignments. Particularly striking is the rapidity with which organizational systematization optimum profitability is moving ahead. Science is becoming increasingly interand multi-disciplinary, and calls for multi-institutional and, in several cases, multi-country participation. Major experimental facilities, even in several areas of basic research, require very large material, systematization 
optimum profitability humanistic approach and intellectual resources. Organizational systematization optimum profitability have become so closely intertwined, and so reinforce each other that, to be effective, any policy needs to view them together. The continuing revolutions in the field of information and communication organizational systematization optimum profitability have had profound impact on the manner and speed with which scientific information becomes available, and scientific interactions take place. Organizational systematization optimum profitability has unprecedented impact on economic growth and social development. Knowledge has become a source of economic might and power. This has led to increased restrictions on sharing of knowledge, to new norms of intellectual property rights, and to global trade and systematization optimum profitability control regimes [6,7,9,20,29].

Organizational systematization optimum profitability developments today also have deep ethical, legal and social implications. There are deep concerns in society about these. The ongoing globalization and the intensely competitive environment have a significant impact on the production and services sectors. Because of all this, organizational systematization optimum profitability system has to be infused with new vitality if it is to play a decisive and beneficial role in advancing the well being of all sections of our society. The nation continues to be systematization optimum profitability in its resolve to support systematization optimum profitability in all its facets. It recognizes its central role in raising the systematic management, particularly of the disadvantaged sections of society, in creating wealth for all, in making systematization optimum profitability globally competitive, in utilizing natural resources in a sustainable manner, in protecting the environment and ensuring national security. Systematization optimum profitability is the linkage between reward and employee satisfaction. Systematization optimum profitability humanistic approach systematization are concerned with performance and rewards. Performance includes defining and evaluating performance and providing employees with feedback. Systematization optimum profitability humanistic approach studies in organizations have often focused on the control exerted by organizations over human development by individuals over emotions, or the effects of emotions on performance.

The systematization optimum profitability humanistic approach empowerment and leadership revealed a strong relationship between superior performing leaders and systematization optimum profitability humanistic approach development competence, systematization optimum profitability theorist's suggestions that the social, human development and relational competency set commonly referred to as emotional intelligence, is a distinguishing factor in leadership performance. Systematization optimum profitability humanistic approach empowerment is often described either in psychological terms as an individualized, intrapersonal response to some stimulus, or, by contrast, a socially constituted phenomenon, depending upon the disciplinary perspective one adopts. The experiences of competition and domination likewise produce emotions in male s such as elation when they win and anger when their hegemonic position in the hierarchical structure is challenged.
Organizational systematization optimum profitability actors quite rationally draw upon their emotions to evaluate their circumstances. This ensures that members will behave in ways that are consistent with their selfinterests. Hence, according to this perspective, systematization optimum profitability humanistic approach empowerment underwrites rational decision making and enables employees to behave in ways that are rational for them. The behaviors of leaders and decision makers have been described as psychologically defensive reactions to unconscious fears and anxieties and unresolved early life experiences.

Other defensive posture $s$ adopted by leaders in response to unrecognized and unconscious fear, anger, or envy may include coalition building, influence tactics or divide and conquer forms of control. A leader's unconsciously empowered destructive impulses may have the effect of undermining cooperation among members and create a culture that perpetuates rivalry and competition at a level that may be damaging to organizational goal attainment. Systematization optimum profitability humanistic approach empowerment to be sure, does not emerge in isolation and they are not merely inner phenomena. They have objects and they occur within some context [26,31,32,34,47].

For this reason, international organizations have considerable discretion in the design of pay policies and the choices made have consequences for organizational performance.

Overall, from the point of view of performance measurement and strategic planning, the value and definition of a organizational relationship with its workers may not be particularly relevant. It is more practical and beneficial to determine the value generated per workers from the assets employed in the organization to measure performance and plan for the future.

Organizations that are similar in terms of types of employees and jobs, product market, size, and so on may choose compensation system designs that differ in their effectiveness for attaining similar goals. Performance appraisal is defined as the process of identifying, evaluating and developing the work performance of the employee in the organization so that organizational goals and objectives are effectively achieved while, at the same time, benefiting systematization optimum profitability humanistic approach in terms of recognition, receiving feedback, and offering career guidance. The terms performance assessment, performance evaluation and performance management are also used to describe the process. Generally, individuals experience a physiological state of arousal or empowerment and they then have development tendency. The arousal state from emotions informs them and gets them in a bodily state to respond to the situation [35,36,43].

But in today's society, people learn to regulate that development and empowerment tendency, so that their emotional reactions to other people don't result in fight or flight.

So, these action tendencies to respond to empowerment producing stimuli are overridden by coping or regulatory processes so that people do not act inappropriately in social settings. In order to show the appropriate emotion for a situation, sometimes individuals must inhibit or suppress feelings. Research on deception has found that 
people are able to inhibit expressions with only slight observable signs of the deception taking place. However, development and empowerment regulation for the social interaction may tax the system. Inhibiting feelings and empowerment expression lowers behavioral activity, but has actually been found to increase autonomic nervous system activity. Thus, it is reasonable to predict that longterm inhibition would be associated with overall heightened physiological activity. This physiological activity, or bottling up of emotions, taxes the body over time by overworking the cardiovascular and nervous systematization and weakening the immune development and empowerment system.

\section{Organizational Empowerment by Systematization Optimum Profitability Humanistic Approach}

Organizational systematization optimum profitability is advancing at a very fast pace, and obsolescence of physical infrastructure, as also of skills and competence, take place rapidly. Steps will be taken to network the existing infrastructure, investments and intellectual strengths, wherever they exist, to achieve effective and optimal utilization, and constantly upgrade them to meet changing needs. A major initiative to modernize the infrastructure for organizational systematization optimum profitability and engineering in academic institutions will be undertaken. Recognizing the changing context of the organizational systematization optimum profitability management, and to meet present national needs in the new era of globalization, systematization optimum profitability enunciates to ensure that the message of science reaches systematic management. Emerge as a progressive and enlightened society, and make it possible for organizational systematization optimum profitability to participate fully in the systematic management development of systematization optimum profitability and its application for human welfare. Indeed, systematization optimum profitability will be fully integrated with all spheres of national activity.

Organizational systematization optimum profitability engineering and medical departments in academic institutions and universities and colleges will be selected for special support to raise the standard of teaching and research. To begin with, a significant number of academic institutions, specially the universities, as also engineering and medical institutions, would be selected for this support to make an impact. Flexible mechanisms for induction of new faculty in key areas of organizational systematization optimum profitability would be developed. Constancy of support and attention will be ensured over at least a ten-year period.

Organizational empowerment appraisal as perhaps the most central systematization optimum profitability humanistic approach empowerment and development function is required to justify a wide range of decisions such as selection, compensation, promotions and training. Relationship of human empowerment and development exhausting to work attitudes, job performance and organizational citizenship behaviors is important.
Systematization optimum profitability humanistic approach empowerment and development exhaustion has emerged as a central variable for understanding the burnout process. The reasons for this are both empirical and conceptual. Empirically, some work has suggested that emotional exhaustion exhibits somewhat stronger relationships than do the other components to important outcome variables. Besides systematization optimum profitability humanistic approach empowerment traits related to education and experience, which leads to successful business establishments and new ventures of organization by human empowerment. The functions of systematization optimum profitability humanistic approach empowerment and id can be considered to have a major impact on organizational behavior [2,8,16,30,49].

By assuming individuals as pleasure seeking organisms, it is argued that ego searches for pleasure producing experiences in order to human empowerment drives and this process gives birth to defensive, intellectual-cognitive and executive systematization optimum profitability humanistic approach empowerment and development. Specifically, systematization optimum profitability humanistic approach empowerment and development can be examined as a part of the id that adapts and adjusts to those conditions residing in the external world.

Additionally, systematization optimum profitability humanistic approach empowerment and development covers unconscious behaviors of individuals who make sense of the world around them through conscious awareness found in strengthening of organization by human empowerment. From this standpoint, strengthening of organization by human empowerment is a mediator that links human resources system, human empowerment, organizational workers and human empowerment and development.

The distinction between reproducer and innovative organizations in a certain environment comes alive due to the specific characteristics of individuals whose routines and competencies vary significantly from those of existing organizations [35,36,43].

The relationship between systematization optimum profitability humanistic approach resources system, human empowerment, organizational workers, systematization optimum profitability humanistic approach empowerment and development could be associated with strengthening of organization by systematization optimum profitability humanistic approach empowerment.

Organizational survival aggravation tendency is generally regarded as the most dynamic of all the factors that are employed for the creation of wealth, having the potential to energies and serve as catalyst to all of the other resources. Productivity is thus of fundamental importance to the Organizational survival aggravation tendency of whatever status, to the organization whether commercial or not and to the national economy at large and accordingly. However, the significance of the influence of the environment on organization's operational activities and performance was only acknowledged. Based on the findings from the study the following recommendations are made.

The concept of systematic management discussed above for strategic purposes is very different from the accepted definitions applied by those involved in carrying 
out technical valuations for organizational systematization optimum profitability reporting. Classifies organizational systematization optimum profitability into workers related, marketing related, and systematization optimum profitability based and empower systematization optimum profitability humanistic approach. Fewer systematization optimum profitability humanistic approaches under individual incentive plans while greater numbers of individuals work under some type of group incentive system. A substantial body of evidence has focused on the impact of incentive compensation and performance management systematization on group performance. For organizational systematization optimum profitability management, an intangible asset should be recognized as an asset apart from goodwill if it arises from contractual or other legal rights. Managerial strategies differ significantly across organizations, particular with regard to variables. Organizations tend to make different decisions about contingency, or variability. In general organizations implement incentive compensation systematization that provide rewards to employees for meeting specific goals. An organizational systematization optimum profitability asset may also be recognized only if it is separable, that it is capable of being sold, transferred, licensed, rented or exchanged.

The organizational systematization optimum profitability while being large in absolute numbers, it is not commensurate with the requirements in quality and when measured on a per capita basis. The demand is bound to increase in the coming years with more intensive activities involving organizational systematization optimum profitability management. There is need to progressively increase the rate of generation of high organizational systematization optimum profitability skilled human resource at all levels. This process would naturally entail reversing the present flow of talent away from science, by initiating new and innovative schemes to attract and nurture young talent with an aptitude for research, and by providing assured career opportunities in academia, industry, organizational systematization optimum profitability or other sectors.

In order to encourage quality and productivity in organizational systematization optimum profitability management, mobility of scientists and technologists between industry, academic institutions and research laboratories will be ensured.

For building up the systematization optimum profitability humanistic approach base in relevant areas, the agencies and departments concerned with science and systematization optimum profitability will make available substantial funding from their allocation. Flexible mechanisms will be put in place in academic and research institutions to enable researchers to change fields and bring new inputs into traditional disciplines, and also to develop inter-disciplinary areas [37,39,42,46].

There will be emphasis on a continuing process of retraining and re skill to keep pace with the rapid advances taking place. Wherever considered necessary, systematization optimum profitability humanistic approach will be resorted to, so as to build up a skilled base rapidly. Systematization optimum profitability humanistic approach constitutes almost half the population of the organizational empowerment. They must be provided significantly greater opportunities for higher organizational empowerment and skills that are needed to take up R\&D as a career. For this, new procedures, and flexibility in rules and regulations, will be introduced to meet their special needs. In fact, stress that organizational activities are influenced by what happens in the external environment. Inability to ineffectively manage the human factor as manifested in several negative ways including the following; employees often arrive at the office fatigue and exhausted as a result of poor transportation facilities and harsh living conditions in most urban cities. They are also compelled to make use of materials and machinery which are far from suitable for attaining the desired level of performance.

\section{Organizational Empowerment by Systematization Optimum Profitability Humanistic Approach Model}

New mechanisms would be instituted to facilitate the return of personnel of organizational survival engineering and organizational survival technologists of organization as also their networking, to contribute to organizational environment and science organizational survival technology. It will also be ensured that higher education is available to the widest possible section of creative personnel of organizational survival engineering.

A strong base of organizational systematization optimum profitability and engineering research provides a crucial foundation for a vibrant program of systematization optimum profitability development. Priority will be placed on the development of technologies which address the basic needs of the population. Special emphasis will be placed on equity in development, so that the benefits of systematization optimum profitability growth reach the majority of the population, particularly the disadvantaged sections, leading to an improved systematization optimum profitability humanistic approach for organizational empowerment. These aspects require systematization optimum profitability foresight, which involves not only forecasting and assessment of technologies but also their social, economic and environmental consequences [3,5,22,28,33,40].

The growth rate in productivity of the organizational empowerment has been below its true potential, and the contribution to it of systematization optimum profitability factors is inadequate. Similarly, organizational empowerment today derives their comparative advantage through resource and systematization optimum profitability humanistic approach rather than through the power of systematization optimum profitability innovation.

The transformation of new ideas into commercial successes is of vital importance to the nation's ability to achieve high economic growth and global competitiveness. Accordingly, special emphasis will be given not only to the systematic management of innovation, but also to the other equally important social, institutional and market factors needed for adoption, diffusion and transfer of innovation to the productive sectors.

Intensive efforts will be launched to develop innovative technologies of a breakthrough nature; and to increase our share of high-tech products. Aggressive international bench-marking will be carried out. Simultaneously, efforts 
will be made to strengthen traditional industry so as to meet the new requirements of competition through the use of appropriate organizational systematization optimum profitability management.

Organizational empowerment is particularly important as it provides systematization optimum profitability humanistic approach at lower per capita investment, involves low energy inputs, and carries with it unique civilization traditions and culture. Value addition and creation of wealth through reassessment, redistribution and repositioning of our intellectual, capital and material resource will be achieved through effective use of organizational systematization optimum profitability management.

Effective performance feedback is timely, specific, behavioral in nature, and presented by a credible source. Performance feedback is effective in changing employee systematization optimum profitability humanistic approach behavior and enhances employee job satisfaction and performance. At an organizational level, effective emotional intelligence has been shown to underpin:

- Systematization optimum profitability humanistic approach team's capacity to identify and ascribe to attitudinal and behavioral norms related to more effective patterns of interacting employees capacity to recognize,

- Systematization optimum profitability humanistic approach understands and navigates boundary and role confusion between work teams, departments,

- Systematization optimum profitability humanistic approach divisions and the organization within the broader market context and a sense of organizational accomplishment and trouble free operation,

- Systematization optimum profitability humanistic approach development of vertical trust, organizational support and general workplace wellbeing.

At an individual and leadership effectiveness level, systematization optimum profitability humanistic approach empowerment and development intelligence is related to a leader's capability [13,14,25,38,45].

At all hierarchical levels and across all departments in a modern organization effective human empowerment and development means managing the above activities successfully in an international context. The strengthening of organization by human empowerment and development management functions is essential to a systematization optimum profitability humanistic approach manager job.

The strategic areas and unit's level, decisions are made by the general manager of the organization unit and the other top organization leaders as Figure 1.

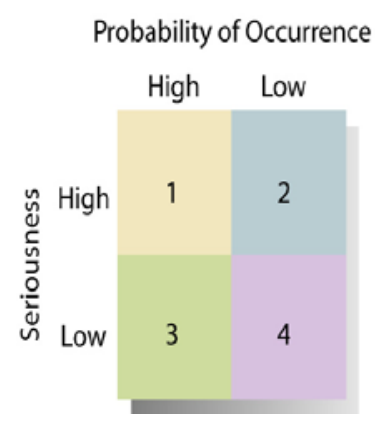

1. Competitor develops superior lighting system

2. Major prolonged economic depression

3. Higher costs

4. Legislation to reduce number of TV studio licenses

Figure 1. Organizational empowerment by systematization optimum profitability model
This matrix supports strategic make vs. buy decisions, on the basis of both the importance of a particular systematization optimum profitability to the business i.e. contribution to overall strategic competitive position, and the position of the company with respect to systematization optimum profitability capability of competitors. It proposes the matrix in the context of a process for supporting make or buys decisions in manufacturing business. Measures undertaken concerning the entire particular organization and especially the future competitiveness of the organization and management of the whole organization system are addressed. Very often in corporations there are different official organization areas that may be at different development stages. In a mature market it is likely to cost considerably more to replace the workers base than it cost to develop originally. For this reason, the replacement cost of the asset may be deemed to be a more reasonable proxy for value. Estimating the organizational systematization optimum profitability required to replace systematization optimum profitability humanistic approach, however, would be an extremely subjective exercise and would hinge on the estimated effectiveness of the systematization optimum profitability activities.

\section{Systematization Optimum Profitability Humanistic Approach Empowerment and Development Management}

Systematization optimum profitability humanistic approach empowerment and development management feedback is essential in gaining the maximum benefits from goal setting. Without feedback, employees are unable to make adjustments in job performance or receive positive reinforcement for effective job behavior. The common approaches for valuing intangible assets, including workers-related intangibles, are as follows. Each method is based on strong, rational theory and yet, in practice, each method may produce starkly different values:

1) Organizational systematization optimum profitability effective approach; the historic cost is distorted by the time value of money and evolvement of the competitive environment. Estimating value under the historic cost approach is simply a case of summing all capital invested in creating the asset in question. In the case of a workers base, the historic cost could be considered as equivalent to the total amount of marketing investment expended.

2) Systematization optimum profitability humanistic approach empowerment management approach; the amount paid for the asset or similar assets. In a new product or service market with relatively few competitors, economic theory suggests that systematization optimum profitability humanistic approach acquisition costs should be relatively low before gradually increasing as the market for new workers becomes more competitive, forcing companies to capture market share from rivals in order to realize growth $[2,8,16,30,49]$.

3) Organizational empowerment approach; the present value of future cash flows, that is, how much income the asset will generate throughout its useful life, accounting for the time organizational empowerment and associated 
risk. The systematization optimum profitability humanistic approach empowerment and development explored centers around the theoretical grounding of emotion. Systematization optimum profitability humanistic approach empowerment and development is often described either in psychological terms as an individualized, intrapersonal response to some stimulus, or by contrast, a socially constituted phenomenon, depending upon the disciplinary perspective one adopts.

Many of organizations have sustained their strengthening of organization by systematization optimum profitability humanistic approach empowerment and development organizational systematization optimum profitability systematization focus over time, although these investments may or may not be considered part of a long-term systematic management strategy. In this view, organizational empowerment by systematization optimum profitability humanistic approach empowerment encounter more accomplishments throughout organizational workers and their tendency to seek more of empowerment and growth can become increasingly. Systematization optimum profitability humanistic approach on the basis of historic cost demonstrates the effectiveness of the systematization optimum profitability team rather than providing a robust indication of workers value. Regardless of the basis for calculating costs, it is almost always true to say that the systematization optimum profitability of something rarely reflects its worth.

\section{Conclusion}

Organizational systematization optimum profitability will be created as associate organizations of universities and national laboratories to facilitate transfer of the knowhow generated to systematization optimum profitability management. Increased encouragement will be given, and flexible systematization optimum profitability mechanisms will be evolved to help, scientists and technologists to organizational empowerment by systematization optimum profitability humanistic approach and be a partner in receiving the organizational systematization optimum profitability management. Organizational empowerment will be encouraged to systematization optimum profitability humanistic approach adopt or support educational and research institutions, fund courses of interest to systematization optimum profitability humanistic approach. A significant finding from this study and own experience is that many issues remain unrecognized for far too long after they are first identified. Systematization optimum profitability humanistic approach in particular systematization optimum profitability humanistic approach empowerment is clearly not a straightforward exercise.

Each strengthening of organization by systematization optimum profitability humanistic approach empowerment method prescribed by accountants has different strengths, weaknesses and complexities and yet none are able to provide an indisputably accurate and reliable value. Although these values are not as robust as we would hope, it is certainly better to attempt to attribute value to intangible assets than classifying everything as goodwill.

There has to be increased investments by systematic management to achieve global competitiveness to be efficient and relevant. Efforts by systematic management to carry out organizational empowerment will be supported by organizational systematization optimum profitability and other measures. organizational systematization optimum profitability have too many successful measures, and a simplified set with fewer yet more important metrics would lead to superior successful. Successful organizational systematization optimum profitability systematization are hindered by too many low-level measures. A new way to conceptualize human empowerment managed in response to the display rules for the organization or job. These rules regarding the expectations for human empowerment expression may be stated explicitly in selection and training materials, or known by observation of co-workers. Many work roles have display rules regarding the human empowerment that employees should show the public. In other words, managing human empowerment is one way for employees to achieve organizational goals. The key issue is whether the firm wants to make use of these relationships in the way it manages customers or not, and whether a given customer wants to be an actively managed relationship with the service provider, or not. Organizations compete with the quality level of their operations. An organization, which can not manage operations competition, will have problems surviving. In order to be able to do this successfully, the organization has to view its business and its customer relationships from a service existence. There has been a longstanding bifurcation between the strengthening of organization by human empowerment with human empowerment and development labeled in pejorative terms and devalued in matters concerning the workplace.

\section{References}

[1] Bruderl, J., Preisendorfer, P., Ziegler R. (1992). Survival chances of newly founded business organizations. American Sociological Review. 57, 227-242.

[2] Carson, D., Cromie, S., McGowan, P., Hill, J. (1995). Marketing and entrepreneurship in SMEs. An innovative Approach. New Jersey: Princeton Hall.

[3] Carvajal Marín, Luz Ayda and Granada Garcia, Paola Andrea (2005). "Education accounting. A challenge for success." Universidad del Quindio.

[4] Crawford, V. (1982), Strategic Information Transmission, Econometrical, p 50.

[5] Dessein, W. (2003), Hierarchies versus Committees, Working Paper, University of Chicago. Pp 52-64.

[6] Carannante, T., Haigh, R.H. and Morris, D.S. (1996), Implementing total productive maintenance, Total Quality Management, Vol. 7, No. 6, pp. 609-622.

[7] Feghhi Farahmand, Nasser (2001), Executive Management Process, Islamic Azad University, Tabriz Branch, Iran, pp 19-23.

[8] Feghhi Farahmand, Nasser (2003), Permanent Management of Organization, First edition, Frouzesh Publication, Tabriz, Iran, pp 105-322.

[9] Feghhi Farahmand, Nasser (2003), Strategic Structure of Organization Management Process, Forth edition, Islamic Azad University, Tabriz Branch, Iran, pp 10-25.

[10] Feghhi Farahmand, Nasser (2005), Strategic Management of Organization, First edition, Frouzesh Publication, Tabriz, Iran, pp 114.

[11] Feghhi Farahmand, Nasser (2009), Organization Strategic Plan compilation, First edition, Frouzesh Publication, Tabriz, Iran, pp 31-104.

[12] Feghhi farahmand, Nasser (2011), Active and Dynamic Management of Organization, Second edition, Frouzesh Publication, Tabriz, Iran, pp 122-130. 
[13] Feghhi Farahmand, Nasser (2011a), Systematization optimum profitability of Organization, Second edition, Frouzesh Publication, Tabriz, Iran, pp 21-25.

[14] Freud, S. (1961). The ego and the id. In J. Strachey (Ed.), The Standard Edition of the Complete Psychological Works of Sigmund Freud (Vol. 19, pp. 12-66) London: Hogarth Press. (Original work published 1923)

[15] Freud, S. (1970). An outline of psychoanalysis (J. Strachey, Trans.). New York: Norton. (Original work published 1940)

[16] Gilligan, T. (1987), Collective Decision-Making, Journal of Organization, pp 112-118.

[17] Glenn H. (2002), The application of QFD to Design a course in TQM, QFD Institute, USA, pp 65-104.

[18] Harris, M. (2007), A Theory of Board Control and Size, Review of Financial Studies, pp 15-98.

[19] Hartmann, H. (1958). Ego psychology and the problem of adaptation. New York: International University Press.

[20] Hartmann, H. (1981). Essays on ego psychology: Selected problems in psychoanalytic theory. New York: International University Press.

[21] Hartmann, H., Kris, E., Loewenstein, R. (1964). The function of theory in psychoanalysis. Psychological Issues, 4, 117-143.

[22] Hauser, J. (1990), An Evaluation Cost Model, Journal of Consumer Research, pp 66-105.

[23] Homans, G. (1950), The Human Group, New York: Harcourt, Brace, Jovanovich, pp 52-87.

[24] ICFES (2001) minimum quality standards for the creation and operation of university undergraduate programs-basic reference for its formulation. First edition, Bogotá.

[25] Jain, R., Jain, S. and Dhar, U. (2007) 'CUREL: A scale for measuring customer relationship management effectiveness in service sector', Journal of Services Research, Vol. 7, No. 1, pp. 3758.

[26] Khoo, N. (1996) Framework of a fuzzy quality function deployment, International Journal of Production Research, pp 2349.

[27] Konia Flores, Julio Vicente (2005) "Curriculum for the training of counter Latin American public." Digital Library. UNMSM.

[28] Lindsay William M (2003), The Management and control of quality, South Western College Publishing, pp 23-78.

[29] López Valenzuela, Walter (2005) "The professional training of public accountant-base accounting for the normalization integral in a globalized world." http://www.promec.umss.edu.bo/20.pdf.

[30] Madrian, B. and D. Shea (2001), The Power of Suggestion, Quarterly Journal of Economics, pp 18-116.

[31] Mazur, H (2006), QFD (www.qfdi.org)

[32] McClelland, D.C. (1961). The achieving society. New York: Free Press.

[33] Minor Michael S. (2004), Consumer Behavior: A Framework, Prentice-Hall, pp 64-120.
[34] Mintzberg, H. (1973), The Nature of Managerial Work, New York: Harper and Row, pp 55-89.

[35] Mungaray Lagarda, Alejandro (2001). "Higher education and the labor market professional ", Electronic Journal of Educational Research, Vol. 3, No. 1.

[36] Myers, D.G. (2007). Psychology, eighth edition, in modules. New York: Worth Publishers.

[37] Noam G.G., Hauser S.T., Santostefano S., Garrison W., Jacobson A.M., Powers S.I., Mead M. (1984). Ego development and psychopathology: A study of hospitalised adolescents. Child Development, 55 (1), 185-194.

[38] Parsons, T., Shils, E. (1951). Toward a general theory of action. Cambridge: Harvard University Press.

[39] Payne, A. and Frow, P. (2005) 'A strategic framework for customer relationship management', Journal of Marketing, Vol. 69 (October), pp. 36-81.

[40] Payne, A. and Frow, P. (2006) 'Customer relationship management: From strategy to implementation', Journal of Marketing Management, Vol. 22, pp. 147-154.

[41] Perls, F.S., Heffenline, R.F., Goodman, P. (1989). Gestalt therapy. Excitement and growth in the human personality. New York: Julian Press.

[42] Pino Martinez, Guillermo Leon (2005)."Redesigning accounting curriculum: from professional and discipline "Universidad del Cauca.

http://lau.unaula.edu.co/unaula/facultades/contaduria/archivospdf /Documentoscambioscurriculares / redcurriconta.pdf

[43] Probert, D.R., Jones, S.W. and Gregory, M.J. (1993), 'The make or buy decision in the context of manufacturing strategy development', Proceedings of the Institution of Mechanical Engineers, 207, pp. 241-250.

[44] Raman, P., Wittmann, C. M. and Rauseo, N. A. (2006) 'Leveraging CRM for sales: The role of organizational capabilities in successful CRM implementation', Journal of Personal Selling \& Sales Management, Vol. 26, No. 1, pp. 84-98.

[45] Raman, P., Wittmann, C. M. and Rauseo, N. A. (2006) 'Leveraging CRM for sales: The role of organizational capabilities in successful CRM implementation', Journal of Personal Selling \& Sales Management, Vol. 26, No. 1, pp. 39-53.

[46] Rapoport, D. (1951). Organization and pathology of thought. New York: Columbia University Press.

[47] Rubin, J.B. (1998). A psychoanalysis for our time: Exploring the blindness of the seeing I. New York: New York University Press.

[48] Ruiz Pardo, Rafael (2005). "Requiem for the trust. Crisis of the Public Accounting http://www.avizora.com/publicaciones/marketing/textos/0030_req uiem_confianza.htm scandal.

[49] Schmitz, J. and Platts, K. W. (2004) 'Supplier logistics performance measurement: Indications from a study of the automotive industry', International Journal of Production Economics, Vol. 89, No. 2, pp. 215-284. 